\title{
Experiencia en la terapia de pacientes pediátricos con Leucemia Linfoblástica Aguda en SOLCA de Manabí.
}

\section{Experience in the therapy of pediatric patients with Acute Lymphoblastic Leukemia at SOLCA de Manabí.}

Terapia y Leucemia linfoblástica aguda .

Richard Cedeño Mera ${ }^{(1)}$

Cecilia Villaprado Meza ${ }^{(2)}$

(1) Hospital Oncológico “Dr. Julio Villacreses Colmont Solca”. Portoviejo, Manabí, Ecuador. email: jefe.oncohemaped@solcamanabi.org

ORCID: https://orcid.org/0000-0003-3731-0822

(2) Hospital Oncológico “Dr. Julio Villacreses Colmont Solca”. Portoviejo, Manabí, Ecuador. email cecivillaprado@hotmail.com

ORCID: https://orcid.org/0000-0002-8274-0904

Contacto: jefe.oncohemaped@solcamanabi.org

\section{Resumen}

Las leucemias se distinguen por infiltración de células neoplásicas del sistema hematopoyético en la médula ósea, sangre y otros tejidos. El objetivo del estudio fue describir la experiencia del Hospital Oncológico "Dr. Julio Villacreses Colmont”). de SOLCA al aplicar la guía de tratamiento del grupo SEHOP- PETHEMA 2013 fundamentada con los últimos avances diagnósticos y terapéuticos desarrollados por el grupo alemán Berlín Frankfurt Muenster (BFM) en pacientes pediátricos con Leucemia Linfoblástica Aguda. Se realizó un estudio de diseño longitudinal con 94 pacientes pediátricos entre 1 y 18 años con diagnóstico de leucemia linfoblástica aguda que recibieron tratamiento con la guía de la Sociedad Española de Hematología y Oncología Pediátrica. Los resultados destacan que de los 94 niños, 54\% pertenecían al género masculino, el rango predominante fue de 2 a 6 años, el inmunofenotipo más frecuente (90\%) fue LLA-B COMÚN, en el estudio citogenético $79 \%$ presento una Diploidia, y 94\% no presentó reordenamientos moleculares, la evaluación de la respuesta a los corticoides en el día +8 fue buena en el $94 \%$ de los niños, en el 
día $+1568 \%$ alcanzaron la remisión completa $(\mathrm{RC}<0,01 \%)$.Se estadificaron 1 paciente en riesgo estándar, 75 en riesgo intermedio y 18 en riesgo alto. Se concluye que la guía SEHOP se puede cumplir eficientemente, sin embargo se deben realizar estudios corporativos a nivel país que permitan identificar nuestras falencias y crear estrategias para optimizar el soporte integral del inmunocomprometido y obtener terapias de salvataje en niños refractarios y recaídos.

Palabras clave: experiencia, pacientes pediátricos, Leucemia Linfoblástica Aguda, SOLCA

\section{Abstracts}

Leukemias are distinguished by infiltration of neoplastic cells of the hematopoietic system into the bone marrow, blood, and other tissues. The objective of the study was to describe the experience of the Oncological Hospital "Dr. Julio Villacreses Colmont"). of SOLCA when applying the SEHOPPETHEMA 2013 group treatment guide based on the latest diagnostic and therapeutic advances developed by the German group Berlin Frankfurt Muenster (BFM) in pediatric patients with Acute Lymphoblastic Leukemia. A longitudinal design study was carried out with 94 pediatric patients between 1 and 18 years of age with a diagnosis of acute lymphoblastic leukemia who received treatment according to the guidelines of the Spanish Society of Pediatric Hematology and Oncology. The results highlight that of the 94 children, 54\% belonged to the male gender, the predominant range was from 2 to 6 years, the most frequent immunophenotype (90\%) was COMMON ALL-B, in the cytogenetic study $79 \%$ presented Diploidy, and $94 \%$ did not present molecular rearrangements, the evaluation of the response to corticosteroids on day +8 was good in $94 \%$ of the children, on day $+1568 \%$ reached complete remission ( $\mathrm{CR}<0.01 \%$ ) 1 patient was staged at standard risk, 75 at intermediate risk, and 18 at high risk. It is concluded that the SEHOP guide can be efficiently complied with; however, corporate studies should be carried out at the country level to identify our shortcomings and create strategies to optimize the comprehensive support of the immunocompromised and obtain salvage therapies in refractory and relapsed children.

Keywords: experience, therapy, pediatric patients, acute lymphoblastic leukemia, SOLCA

\section{Introducción}

La leucemia linfoblástica aguda (LLA) es consecuencia de la transformación maligna de una célula progenitora linfoide inmadura que tiene la capacidad de expandirse y formar un clon de células progenitoras idénticas bloqueadas en un punto de su diferenciación. Es la neoplasia más frecuente 
en la infancia, constituyendo el $80 \%$ de todas las leucemias agudas de la edad pediátrica (Atienza, A. L.2016).

El grupo internacional del Berlín Frankfurt Muenster (BFM) original ha desarrollado varios estudios clínicos exitosos, todos derivados en su estudio inicial ALL IC -BMF 2002, basado en los resultados de este importante grupo la Sociedad Española de Hematología y Oncología Pediátricas (SEHOP) y el Programa Español de Tratamientos en Hematología (PETHEMA) desarrollo la guía de tratamiento SEHOP - PETHEMA - 2013 para niños entre 1 y 18 años mismo que en Hospital Oncológico “ Dr. Julio Villacreses Colmont” SOLCA Portoviejo paralelamente también hemos venido utilizando desde el año 2013.

Los grupos internacionales más reconocidos, históricamente han obtenido de forma ascendente y paulatina mejores resultados de supervivencia libre de enfermedad a 5 años como por ejemplo $>$ al 75\% BFM 90: 78, Cancer Children Group: CCG 1800 75\%, German Cooperative Study Group for ALL: COALL 92 77\%, Dana Farber Cancer Institute: DFCI 91 83\%, Nordic Society of Pediatric Haematology and Oncology: NOPHO III 78\%, St. Jude Children's Research Hospital: SJXIIIB $81 \%$ ). Hasta en el momento actual en que todos estos grupos de trabajo han alcanzado supervivencias libres de enfermedad superiores al $90 \%$ y en el que todos confluyen en el uso esquemas de poliquimioterapia y estratificación de sus enfermos de acuerdo a los resultados de la medición de la EMR por biología molecular o por citometría de flujo (Agriello, et. al., 2017).

Existen interesantes estudios, que corroboran la realidad antes mencionada, pero aun en todo este contexto es sorprendente que ante una patología tan importante como la leucemia linfoblástica pediátrica los países en vías de desarrollo no existen suficientes estudios y análisis de datos sobre esta y sus comportamiento y eficacia tras la aplicación de esquemas de tratamiento perfectamente establecido a nivel mundial.

En Ecuador también aplicamos guías de tratamiento internacionales y a díe de hoy ofrecemos esquemas terapéuticos semejantes a los que se realizan a nivel internacional sin embargo no conocemos de manera precisa los resultados en todos los hospitales donde se trata niños con Leucemia Linfoblástica, carecemos de registros que plasmen fehacientemente la experiencia en el uso de estas guías basadas en técnicas especiales de diagnóstico y esquemas de poliquimioterapia que en el devenir de la evolución de nuestras estructuras hospitalarias hemos adquirido y ahora podemos ofrecer con todas sus variables de enorme complejidad. 
No existen los datos suficientes que expresen la realidad de nuestro país y no hemos podido compilar, analizar nuestros resultados para darlos a conocer, la razones son innumerables empezando por las limitaciones en la educación, formación y adquisición de hábitos de investigación, sumado a los sistemas laborales que priorizan el trabajo medico remunerado por atención directa al paciente y el poco estímulo y retribución que tiene la investigación en el ámbito hospitalario, y en otras ocasiones no se poseen los recursos técnicos y tecnológicos que componen los protocolos y que permiten un seguimiento correcto de la enfermedad en su diagnóstico, evolución, tratamiento y pronóstico de la enfermedad.

Es importante señalar que una clave en los esquemas de tratamiento contra la LLA es la utilización de técnicas especiales como la citometría de flujo, la citogenética y la biología molecular que facilitan y precisan el correcto diagnóstico, estratificación de los pacientes en diferentes grupos de riesgo junto con las características clínicas y biológicas del paciente y de la leucemia, así como evaluación de la respuesta temprana a la terapia (López-Hernández, G. 2020).

A nivel nacional existen trabajos que empleando este tipo de guías y cumpliendo las características de diagnóstico, seguimiento y terapia específica expresan supervivencias a 5 años entre un $55 \%$ y 65\% según grupos de trabajo de los diversos hospitales oncológicos de las principales ciudades del Ecuador donde de manera individual desarrollan diversas guías según la formación y experiencia adquirida en el centro donde se formaron sus respectivos líderes, si bien es cierto estos regímenes actualizados son también muy similares y se basan en combinaciones de fármacos oncológicos (Bonilla García, C. D. 2019).

El hospital Oncológico "Dr. Luis Villacreses Colmont" SOLCA de Portoviejo es el centro de referencia de Manabí, la tercer provincia en población del Ecuador, que además recibe pacientes de otras provincias como Esmeraldas, Santo Domingo y Santa Elena desde su fundación en el año del 2005 no cuenta con ningún estudio analítico ni descriptivo de su actividad en el campo de la leucemia aguda Linfoblástica pediátrica, por esta razón sin ningún dato precedente se decidió desde agosto del 2013 recopilar los datos de los niños oncohematológicos y analizar la experiencia de este importante centro de referencia.

Realizar este estudio representa un importante punto de partida que nos permitirá desarrollar más trabajos de investigación al tener una base real de registro de pacientes de SOLCA Portoviejo a través del cumplimiento estricto de la guía de tratamiento de la leucemia infantil SEHOPPETHEMA - 2013, que se basa en todas las evoluciones históricas de desarrollo del protocolo del 
BFM. Toda vez que se han estandarizados todos los lineamientos necesarios y existe el acceso a la información veraz de los estudios diagnósticos.

En este orden debemos destacar la importancia de tener a nuestro alcance de manera ágil y sistemática el estudio de la enfermedad mínima residual a través de la citometría de flujo técnica que permite diagnosticar precisamente y estratificar a los pacientes de en grupo de riesgo y de esta manera intensificar y direccionar el mismo según dicha división.Los resultados de la enfermedad mínima residual por citometría de flujo nos permiten modular la terapia e inclusive predecir la evolución y el pronóstico de la enfermedad.

Previo al 2103 no existen datos hospitalarios que muestren todos los diagnósticos, los grupos de riesgos, la respuestas y el seguimiento preciso de la enfermedad en cada niño como enmarcan las guías de tratamiento, las medidas de bioseguridad no estaban regladas y estandarizadas, el acceso al soporte transfusional y todas las pruebas diagnósticas de la propia enfermedad así como sus controles y las pruebas generadas de las complicaciones infecciosas eran solventadas económicamente por sus familiares al igual que su logística y gestión. Tras un importante cambio en las dinámicas organizativas y el trabajo conjunto de autoridades médicos y familiares se ha logrado un vuelco de 180 grados soslayando todas las adversidades comentadas.

Enmendadas todas estas variables que también impedían un correcto cumplimento de la guía y el reclutamiento de los niños y sus datos se pudo emprender este trabajo que de manera preliminar nos permitirá establecer datos demográficos y evidencia de respuesta que en futuros trabajos nos lograran conocer tasas de remisión, supervivencia global, supervivencia libre de enfermedad y finalmente contrastar con los resultados que existen en la literatura.

Una vez logrado lo anterior, se podrá comparar con los resultados en países de primer mundo donde también aplican estas guías y modelos de atención, todo lo cual permitirá definir y corregir las variables que impiden que estos protocolos que a día de hoy tienen porcentajes de supervivencia superiores al 90\% de curación, no tengan los mismos resultados en nuestros hospitales.

El presente estudio tiene como objetivo, exponer la experiencia del Hospital Oncológico “Dr. Julio Villacreses Colmont"). de SOLCA al aplicar la guía de tratamiento del grupo SEHOP- PETHEMA 2013 fundamentada con los últimos avances diagnósticos y terapéuticos desarrollados por el grupo alemán Berlín Frankfurt Muenster (BFM) en pacientes pediátricos con Leucemia Linfoblástica Aguda. 


\section{Material y Métodos}

La investigación corresponde a un estudio de corte longitudinal que comprende el periodo de agosto de 2013 hasta agosto de 2018. Tiempo en que se ha logrado compilar un numero representativo de niños con leucemia Linfoblástica que nos permite tener una visión objetiva del cumplimiento de las condiciones de las guías, las dificultades técnicas en su aplicación y la efectividad del mismo.

En este sentido, es válido destacar que responde a un enfoque mixto y al análisis de las variables: cantidad de niños, edad, género, procedencia geográfica, factores genéticos, moleculares y de respuesta a la terapia. Para la concreción y logro del objetivo de la investigación se aplicaron métodos del nivel teórico: análisis - síntesis, inductivo - deductivo e histórico - lógico, que permitieron incursionar en el objeto de estudio relacionado con la experiencia en la terapéutica de pacientes pediátricos con leucemia linfoblástica aguda en SOLCA de Portoviejo.

Desde los métodos empíricos se utilizaron: la revisión documental con énfasis particular en las historias clínicas de los pacientes que forman parte de la muestra de 94 niños y las bases de datos que contienen evaluaciones de los resultados emitidos por instituciones externas (Laboratorio Cytolab) que en su quehacer son aliadas estratégicas de SOLCA para el diagnóstico de los niños.

Los datos recopilados fueron procesados por el programa estadístico SPSS, en coordinación con investigadores de la Universidad Técnica de Manabí. Los resultados tienen una alta valía, confiabilidad y se aplicó bajo el consentimiento informado a los padres de los niños.

También se empleó la observación como método científico por excelencia, que develó el estado clínico de los pacientes, la evolución y el criterio final en la respuesta al tratamiento siempre sustentado por los estudios biológicos que se hayan enmarcados en las guías y en el conocimiento actual del seguimiento de los niños con hemopatías malignas. Vale destacar que como investigadores se ha pautado la devolución ética de los resultados a los padres de los niños que formaron parte de la muestra.

\section{Resultados}

La investigación corresponde al periodo de agosto de 2013 hasta agosto de 2018, fueron incluidos 94 pacientes con Leucemia Linfoblástica Aguda, de los cuales 51 pacientes corresponden el 54\% al género masculino y 43 pacientes al $46 \%$ del género femenino. 
El estudio incluye pacientes entre 1 y 18 años, que distribuidos por rango etario corresponden 43 niños (46\%) de 2 a 6 años, 29 adolescentes (31\%) entre 12 a 18 años, 17 escolares (18\%) entre 6 a 11 años y 5 lactantes mayores (5\%) de 12 y 23 meses.

La mayoría de la población en estudio provienen de la ciudad de Portoviejo, seguido por la ciudad de Manta y de Chone.

Respecto al inmunofenotipo los 85 casos más frecuentes (90\%) es el de leucemia Linfoblastica B común (LLA-B COMÚN) sin embargo, se observan 5 casos (5\%) de Leucemia Linfoblástica Pre B (LLA PRE-B) 3 (3\%) casos de Leucemia Linfoblastica Pro B (LAL-PRO+B) y un (1\%) 1 caso de Leucemia Linfoblástica T. (LAL-T) Si relacionamos la estirpe con la edad, identificamos que el mayor número de niños pertenece al fenotipo de LLA-B COMÚN y cuantitativamente se presenta predominantemente en el grupo etario de 2 a 6 años (46\%), seguido del grupo adolescentes (31\%).

En cuanto al diagnóstico citogenético, 74 pacientes (79\%) presentaron Diploidia, 7 pacientes (8\%) representan a Hiperdiploidia alta, $5(5 \%)$ hiperdiploidia baja, $5(5 \%)$ hipodiploida , 2 (2\%) casi tetraploidia y $1(1 \%)$ baja hipodiploidia.

Al realizar el estudio de biología molecular revela que, 88 pacientes (94\%) no presentaron reordenamientos moleculares, 4 pacientes (4\%) presentaron alteraciones moleculares de buen pronóstico y 2 pacientes (2\%) con alteraciones moleculares de mal pronóstico.

La primera evaluación que se realiza en la fase de inducción de la guía es la respuesta a los corticoides en el día +8 de la terapia día en la que 88 pacientes $(94 \%)$ tienen una buena respuesta a los esteroides (menos de 1000 blastos en sangre periférica); la segunda evaluación es la enfermedad mínima residual ( EMR) en del día 15 a través de la citometría de flujo en este estudio 64 pacientes (68\%) alcanzaron la remisión completa $(<0,01 \%), 18(19 \%)$ no remisión $(0,01$ - 10) y 12 pacientes $(13 \%)$ con mala respuesta $(>10)$.

Con la evaluación precedente y juntos con los restantes datos biológicos sumados a las evidencias clínicas como la edad, numero de leucocitos al diagnóstico se logra estratificar a los infantes en grupo de riesgos obteniendo la siguiente división. Riesgo estándar 1 paciente (1\%), Riesgo intermedio 75 pacientes $(80 \%)$, y riesgo alto 18 pacientes $(19 \%)$.

La última y más importante evaluación de la respuesta al tratamiento se realiza en el día + 33 a través de la enfermedad mínima residual es fundamental este resultado en el devenir terapéutico y pronostico del paciente, los resultados revelan que a este día excluyendo los 7 exitus letalis 
acontecidos en este primer mes llegaron 87 casos, los de los cuales 82 niños (89\%) alcanzaron remisión completa $(<0,01), 4(4 \%)$ con mala respuesta $(>1)$ y $1(1 \%)$ en remisión.

De aquellos pacientes que no alcanzaron la remisión completa en el día +33 se deben realizar un estudio de EMR en el día + 52, esta evaluación se realizó solo en 2 pacientes y ninguno de ellos alcanzo el estatus de enfermedad no detectable.

Al finalizar la fase aguda e intensiva de la guía es decir previo a iniciar la fase de mantenimiento de los 94 pacientes que iniciaron el estudio solo 75 pacientes alcanzaron la remisión completa.

Al completar los 2 años tratamiento estandarizado es decir al final del mantenimiento 67 pacientes llegaron a remisión completa, y 8 pacientes recayeron en este periodo de tiempo y todos los cuales fallecieron durante esta fase.

$\mathrm{Al}$ año de fin de tratamiento 66 pacientes llegaron a remisión completa, 1 paciente en recaída el cual también falleció.

\section{Discusión}

Se ha evidenciado que en el grupo estudio la edad de mayor prevalencia para la presentación de la leucemia Linfoblástica pediátrica es la comprendida entre 2 y 6 años de edad con un $46 \%$, datos que coinciden totalmente con la literatura mundial en la cual el rango etario de la LLA pediátrica corresponde a este grupo de edad. Eso se corrobora en diversos estudios conocidos como es el caso del desarrollado el grupo cubano (García et. al.,2017) donde expresan que la mayor prevalencia de sus pacientes pertenece al grupo de 1 a 4 años de edad, por tal motivo coincidimos que el grupo etario predominante es comprendido en la edad preescolar.

De la misma manera el fenotipo más frecuente de leucemias Linfoblástica agudas encontrado entre nuestros pacientes es el B COMUN, dato que coincide totalmente con toda la literatura conocida en cuanto a la estirpe más habitual de la célula linfoide tumoral, concordando con varios autores (Ontario 2016)

El estudio citogenético de la célula maligna en muchos pacientes expresan ganancias perdidas y translocaciones de sus cromosomas lo cual permite caracterizar mejor a la enfermedad y descubrir alteraciones que no solo ayudan en el diagnostico si no también en el tratamiento y pronóstico de la hemopatía, por tal motivo el presente estudio revelo que 74 pacientes (79\%), presentaba diploidía es decir sin alteración citogenética con un cariotipo 46XY ( XX) y solo 20 pacientes (21\%) presento 
ganancias con hiperdiploidias y perdidas con hipodiploidias. Estos datos son variables con los diversos reportes de la comunidad científica, pero pueden ser correspondientes a nuestra variabilidad racial y la genética implícita de esta manera autores como (López 2020) expresan que la alta hiperdiploidía (> 50 cromosomas por célula leucémica) ocurre en $25 \%$ de los niños con Leucemia Linfoblástica Aguda.

Uno de los puntos de mayor relieve de evidencia de cumplimiento de la guía, así como de las capacitaciones técnicas y tecnológicas que a día de hoy poseemos en el control de enfermedad mínima residual con la citometría, misma que queda reflejada en sistemático control en los puntos de evaluación que a continuación se develan. En la evaluación de la EMR en el día +15 a través del reconocimiento del fenotipo maligno inicial se observó que 64 pacientes (68\%), alcanzaron la remisión completa $<0,01 \%$, situación que concuerda con los estudios conocidos en el análisis de respuesta de la medula ósea en el Ecuador de la guía como ejemplo del mismo (Soria 2020)

Cuando conjuntas todos los datos clínicos del debut de la enfermedad como son la edad del niño, el número de leucocitos al diagnóstico, y los parámetros biológicos ya conocidos más las respuestas en los puntos de evaluación se logra establecer los grupos de riesgo como marca la guía. En lo que compete a la evaluación de la EMR destacamos que los puntos de corte para dicha estratificación están basados en la propuesta por el grupo Italiano AIEOP (2018) que los definió en 3 grupos respectivamente según la cuantificación del porcentaje de numero de blástos a través de la citometría: Estándar $(<0,1 \%)$, intermedio $(0,1 \%-<10 \%)$ y alto $(\geq 10 \%)$ Tomando en cuenta todos los aspectos mencionados el grupo de niños del estudio en concordancia con lo indicado se estratificaron a los infantes en grupo de riesgo estándar 1 paciente, riesgo intermedio 75 pacientes, y en riesgo alto 18 pacientes, datos que coinciden correspondientemente con las publicaciones al respecto donde se señala que el grupo más grande de estos es el intermedio.

La segunda evaluación por citometría de la inducción en el día +33 es un dato de enorme importancia en el futro de las estrategias a seguir para la curación del paciente o de la necesidad de requerir un transplante de progenitores hematopoyéticos que permita consolidar la curación del niño, los datos compilados indican que en este día 82 niños alcanzaron remisión completa $(<0,01)$ al confrontar estas cifras con las conocidas en el ambiente de la Oncohematología pediátrica observamos que la revisión de los artículos confirma tasas de respuesta superiores en este día en países de primer mundo situación que está relacionada con la terapia de soporte, sistemas de bioseguridad, pruebas de diagnóstico microbiológico y el acceso farmacológico de medicamentos de alto coste. 
La Leucemia aguda linfoblástica por definición es una enfermedad impredecible tanto en el diagnóstico y evolución, no es posible precisar el momento exacto en que una célula blastica hace el stop madurativo e inicia la proliferación exponencial, pese a ello los avances en el estudio y conocimiento de la enfermedad han logrado a día de hoy alcanzar tasas de supervivencia de hasta el 95\% en países desarrollados.

Pese la utilización de las mismas guías farmacológicas internaciones en países en vías de desarrollo no se alcanzan tasas de supervivencia que superan el $70 \%$ pese a los avances logrados en los últimos años en materia de infraestructura de las áreas, medidas de bioseguridad, métodos diagnósticos de la enfermedad y microbiológicas, a la vista de los resultados se deberá continuar optimizando del proceso y sobre todo lograr vehiculizar los accesos a fármacos y procedimientos de mayor coste y complejidad para los pacientes que no alcanzan remisión completa y que son los que inciden de forma gravitante para no alcanzar los objetivos de supervivencia planteados.

El equipo de Oncohematología pediátrica y las autoridades de SOLCA-Portoviejo en los últimos 7 año han hecho todos los esfuerzos para lograr la mejor estratificación de riesgo, la interacción directa con otras técnicas, vigilancia de la enfermedad mínima residual y la administración de terapia específica dirigida, obviamente fundamentado por la cohesión del grupo profesional multidisciplinario sensibilizado solidario y humano que junto a la abnegación, sacrificio y amor de los padres y familiares del niño oncohematológico son los argumentos fundamentales para seguir brindando ese hilo indestructible de esperanza de vida.

\section{Conclusiones}

Como se ha demostrado en la literatura científica mundial las técnicas de diagnóstico de hematología especializada han sido fundamentales para mejorar el diagnóstico, tratamiento y seguimiento de pacientes con LLA, por tal motivo en el trabajo realizado la citometría de flujo, citogenética y biología molecular, fueron una herramienta útil, sensible y específica, que permitió identificar, caracterizar y cuantificar la presencia de pequeñas o grandes clonas de células tumorales en todos los pacientes del estudio.

Se ha objetivado la guía SEHOP-PETHEMA 2013 la cual se pudo cumplir eficientemente, sin embargo se necesitan estudios cooperativos a nivel nacional que permitan definir el perfil genético de respuestas, las carencias farmacológicas y de diagnóstico microbiológico y así mismo crear estrategias para optimizar el soporte integral del inmunocomprometido y obtener terapias de salvataje en niños refractarios y recaídos creando vías de acceso a fármacos de última generación, 
procedimientos de alta complejidad como el Trasplante de progenitores hematopoyéticos y terapia genética.

\section{Bibliografía.}

Agriello, E., Cazap, N., Dourisboure, R., Fernández, I., Ferrari, L., \& Fishman, L. (2017). Leucemias agudas. Sociedad Argentina de Hematología, 329-358.

Atienza, A. L. (2016). Leucemias. Leucemia linfoblástica aguda. Pediatr. Integr, 20(6), 380-389.

Bartram, J., O’Connor, D., Enshaei, A., \& Moorman, A. (2019). Combining Genotype. Profiling with MRD for More Accurate Prognostication in Acute Lymphoblastic Leukemia. Clinical Lymphoma, Myeloma and Leukemia, 19, S63-S65.

Béné, M. C., \& Eveillard, M. (2018). Evaluation of minimal residual disease in childhood all. International journal of laboratory hematology, 40, 104-108.

Berry, D. A., Zhou, S., Higley, H., Mukundan, L., Fu, S., Reaman, G. H., ... \& Radich, J. P. (2017). Association of minimal residual disease with clinical outcome in pediatric and adult acute lymphoblastic leukemia: a meta-analysis. JAMA oncology, 3(7), e170580-e170580.

Bonilla-García, C. D. (2019). Análisis de supervivencia de los pacientes con diagnóstico de leucemia linfoblástica tratados Hospital Oncológico Solón Espinosa Ayala (SOLCA) núcleo Quito en el período 2000-2017.

Campana, D., \& Pui, C. H. (2017). Minimal residual disease-guided therapy in childhood acute lymphoblastic leukemia. Blood, The Journal of the American Society of Hematology, 129(14), 1913-1918.

García, S. R., Pérez, A. M., Ávila, M. H., Figueras, Y. S., Yara, C.., de Ávila, C., \& de Ávila, P. N. C. Leucemia Linfoblástica Aguda en edades pediátricas, un reto para el Primer Nivel de Atención en Salud.

Gupta, S., Devidas, M., Loh, M. L., Raetz, E. A., Chen, S., Wang, C., ... \& Dunsmore, K. P. (2017). Flow cytometric vs morphologic assessment of remission in childhood acute lymphoblastic leukemia: A report from the Children's Oncology Group (COG).

Leukemia. Health Quality Ontario. (2016). Minimal residual disease evaluation in childhood acute lymphoblastic leukemia: an economic analysis. Ontario health technology assessment series, $16(8)$

López-Hernández, G. (2020). Acute lymphoblastic leukemia. Genetic mechanisms. Revista de Hematología, 20(4), 273-277 
O'Connor, D., Moorman, A. V., Wade, R., Hancock, J., Tan, R. M., Bartram, J., \& Hough, R. (2017). Use of minimal residual disease assessment to redefine induction failure in pediatric acute lymphoblastic leukemia. Journal of Clinical Oncology, 35(6), 660 - 667.

Petit, A., Trinquand, A., Chevret, S., Ballerini, P., Cayuela, J. M., Grardel, N., \& Thouvenin, S. (2018). Oncogenetic mutations combined with MRD improve outcome prediction in pediatric T-cell acute lymphoblastic leukemia. Blood, 131(3), 289-300.

Sánchez, L. M. M., Jaramillo, L. I. J., Hernández, L. F. Á., Restrepo, F. H., Mejía, C. R., \& Álzate, J. D. V. (2018). Enfermedad Mínima Residual en leucemia: rompiendo el paradigma de remisión completa. Revista Cubana de Hematología, Inmunología y Hemoterapia, 34(1), 21 32.

Soria, M., Ferraro, C., Morán, L., Gutiérrez, M., Prada, S., Gaillard, M., \& Drelichman, G. (2020). Leucemia linfoblástica aguda pediátrica, evaluación de enfermedad mínima residual. Revista Hematología, 24(2), 1-1.

Theunissen, P., Mejstrikova, E., Sedek, L., van der Sluijs-Gelling, A. J., Gaipa, G., Bartels, M., ... \& Buracchi, C. (2017). Standardized flow cytometry for highly sensitive MRD measurements in B-cell acute lymphoblastic leukemia. Blood, 129(3), 347-357. 